Service social

\title{
De la pratique intégrée en travail social au Québec : une analyse des principales manifestations
}

\section{Marcelle Laforest}

Volume 36, numéro 2-3, 1987

Approches intégrées

URI : https://id.erudit.org/iderudit/706360ar

DOI : https://doi.org/10.7202/706360ar

Aller au sommaire du numéro

Éditeur(s)

École de service social de l'Université Laval

ISSN

1708-1734 (numérique)

Découvrir la revue

Citer cet article

Laforest, M. (1987). De la pratique intégrée en travail social au Québec : une analyse des principales manifestations. Service social, 36(2-3), 213-226. https://doi.org/10.7202/706360ar
Résumé de l'article

L'émergence de pratiques sociales intégrées au Québec traduit un mouvement de recherche de synthèse au sein du travail social. La théorie des systèmes a joué ici un rôle important dans la convergence des connaissances en travail social. Dans le même esprit, on observe un effort renouvelé dans l'articulation des méthodes de service social et dans des synthèses idéologiques touchant les pratiques sociales intégrées. 
LAFOREST, Marcelle, professeure à l'École

de service social de l'Université Laval.

\section{De la pratique intégrée en travail social au Québec : une analyse des principales manifestations ${ }^{1}$}

\section{Marcelle Laforest}

Dans le champ du travail social, qu'il s'agisse des lieux de la pratique ou de ceux de la formation, on se préoccupe depuis longtemps de la mise en place d'une approche qui puisse s'appliquer aux différentes situations d'intervention que les travailleurs sociaux rencontrent dans leur travail quotidien. Dès 1917, Mary Richmond, première théoricienne du travail social, en affirmait les bases communes et s'élevait contre les divisions, tant au niveau des méthodes de service social qu'à celui des champs de pratique.

«Pour l'essentiel, les méthodes et les buts du casework devraient être les mêmes dans tous les différents services [...] Évidemment, certaines procédures pourraient s'appliquer particulièrement à un ensemble de cas, et d'autres à un ensemble différent, selon la difficulté spécifique considérée. Mais les choses qu'on a le plus besoin de dire à propos du casework sont celles qui sont communes. La division du travail social en des secteurs et des spécialités a été à la fois quelque chose de pertinent et une nécessité. Des ressemblances fondamentales demeurent, cependant. $~_{2}$

La littérature en travail social est jalonnée d'affirmations et de mises en garde de ce genre. Ainsi, plus récemment, en 1977, un Américain, Harry Specht, et une Anglaise, Anne Vickeray ont édité un ouvrage portant sur l'intégration des méthodes en travail social. ${ }^{3}$ Après avoir introduit la recherche d'une approche unitaire comme une "quête à caractère endémique » dans l'histoire du travail social, Specht et Vickeray ont entrepris de faire, avec la collaboration d'une dizaine d'autres auteurs, une synthèse des développements récents de la pensée unificatrice 
en travail social. De même, au Québec, dans un article publié en 1984, Francine Ouellet, ${ }^{4}$ cherchant "à retracer historiquement les éléments qui ont questionné la pratique comme entité $[\ldots]$ ". 5 , en relève plusieurs qui lui permettent de repérer, en divers lieux, à diverses époques et sous diverses plumes, des efforts de "conceptualisation de la pratique du service social comme méthode unitaire ${ }^{\prime} .6$

Dans les milieux de la pratique, il est également rapporté, à diverses époques, des expériences, dites pilotes, d'intervention intégrée. À titre d'exemple, mentionnons celle citée pour la première fois en 1965 et souvent reprise par la suite de "The House on Sixth Street "à New York, 7 comme un exemple-type d'approche unifiée. Vers la fin des années 1970-début 1980, au Québec, l'expérience d'approche intégrée menée par l'équipe d'intervenants sociaux de la Malbaie du Centre de services sociaux de Québec, ${ }^{8}$ nous en ont fourni une illustration particulièrement intéressante. Issu de ce projet-pilote, un regroupement de praticiens sociaux de diverses régions du Québec, dit Groupe d'échange sur les approches intégrées (le GEAl), a vu le jour en 1984. Ce regroupement s'intéresse particulièrement à l'unification des diverses approches de la pratique sociale et ce, dans un but de ressourcement professionnel et de renouvellement des pratiques.

Aussi sommaire soit-il, ce rapide exercice de repérage de tentatives d'intégration des pratiques dans l'histoire du travail social nous confirme la persistance du mouvement. Aussi pourrions-nous croire, à première vue, qu'il s'agit là simplement d'une idée qui fait son chemin comme une contribution parmi d'autres à l'évolution du travail social. Or, tel ne semble pas être tout à fait le cas, puisque les préoccupations d'hier et celles d'aujourd'hui à ce sujet, nonobstant les formes empruntées et le focus retenu selon les époques, demeurent sensiblement les mêmes. D'autre part, ce questionnement nous paraît, de prime abord, si judicieux d'une époque à l'autre que l'on est porté à se demander pourquoi l'avènement de l'approche unitaire est encore pour demain. Plus! comment se fait-il que, loin d'avoir donné lieu à l'approche intégrée tant convoitée, toutes ces préoccupations et remises en question aient plutôt abouti à la production de plusieurs approches qui se disent intégrées, mais qui, paradoxalement, semblent ajouter à la différenciation plutôt que de la réduire.

Ainsi donc, l'évolution des connaissances et des idées, et partant de la pratique en travail social, ne semble pas suffisante pour expliquer ce mouvement d'intégration qui accompagne le cheminement du travail social tout au long de son histoire, mouvement dont les variations semblent dues plus aux particularités des conjonctures et des époques qu'à un progrès réel du phénomène lui-même. Comment s'explique-t-il 
alors et quel rôle joue-t-il dans le développement du travail social ? C'est l'objet même de cet article que de tenter de répondre à cette question. Nous proposons d'abord une hypothèse d'exploration reposant sur le rapport différenciation/intégration, que nous considérons comme étant à l'œuvre dans tout champ de travail. Cette proposition nous ouvrira la voie à la mise en relief des mécanismes qui nous semblent mis à contribution dans le processus d'évolution des pratiques du travail social. Cet exercice apportera, nous l'espérons, un éclairage supplémentaire sur le sujet.

\section{Une proposition de départ}

Notre hypothèse prend comme point de départ le postulat, admis en sciences sociales, selon lequel toute différenciation (au sens de complexification des opérations, de spécialisation des tâches, de division des activités et des responsabilités, etc.) que connaît un champ de travail, qu'elle soit recherchée ou non, conduit inévitablement à des tensions et des menaces de rupture entre ses éléments constitutifs. En réaction à ces perturbations, que d'aucuns nomment dysfonctions ou encore malaises ou crises et qui risquent de faire éclater ce champ, il émerge fatalement des tentatives qui, ou bien en consolident les mécanismes de régulation ou de contrôle déjà existants, ou bien, si ces derniers s'avèrent insuffisants, cherchent à en engendrer de nouveaux. Ainsi, le recours à des mécanismes d'intégration permet-il au champ de s'adapter à de nouvelles conditions et ainsi de procéder à son développement tout en assurant la cohésion nécessaire à son maintien et, partant, à sa survie. En somme, ce processus porteur de changement est en soi une source de désorganisation pour le champ en question, dont la survie, l'identité et la légitimité seraient menacées si la cohésion et l'intégration entre les éléments qui se différencient n'étaient pas assurées.

Ainsi donc, toute opération de différenciation structurelle - s'agissant de la structure du champ de travail - serait flanquée de la mise en œuvre de mécanismes d'intégration et de coordination dont la fonction principale serait d'assurer le maintien du champ pendant que ce dernier tente de gérer les pressions transformatrices. Est-ce à dire que la nature des pressions de changement influencerait la nature des mécanismes d'intégration? La vision mécaniciste a quand même ses limites lorsqu'appliquée au changement social. Nous croyons néanmoins que le rapport entre les deux dimensions nous offre une voie propice à la compréhension et à l'analyse de l'évolution de la pratique sociale et, partant, du travail social. 
Nous posons donc comme proposition de départ que l'émergence de pratiques sociales intégrées au Québec - qu'elles se réclament de l'intitulé "approche intégrée " ou qu'elles en possèdent les attributs traduit un mouvement de recherche, au sein du champ du travail social, de la convergence et de la synthèse d'éléments constitutifs et d'éléments différenciés. Cette recherche emprunte les mêmes voies par lesquelles certains éléments du champ se sont différenciés, à savoir: les voies théorique, méthodologique et idéologique. Dans cette optique, les pratiques sociales intégrées participent à la (re)constitution d'une identité plurielle du travail social, comme est lui-même pluriel le procédé emprunté pour ce faire.

L'analyse que nous nous proposons de faire, ci-après, n'a d'autre intention que de garantir des fondements empiriques à notre proposition. Pour ce faire, nous allons tenter de retracer ces fondements d'ordre théorique, méthodologique et idéologique dans des écrits récents de quelques auteurs québécois en travail social, écrits que nous considérons comme étant porteurs de ce mouvement.

\section{La théorie des systèmes dans la recherche de la convergence des connaissances en travail social}

Déjà en 1972, Jeannine Godbout, dans un examen des «nouveaux défis de la pratique professionnelle " ${ }^{9}$ constate, entre autres, " le caractère périmé de certains processus traditionnels d'interprétation et d'intervention " 10 et " l'émergence d'un pluralisme tant idéologique qu'analytique et méthodologique pour la pratique professionnelle d'aujourd'hui ». ${ }^{11}$ Au terme de sa réflexion, l'auteure entrevoit un avenir prometteur à la théorie des systèmes, de même qu'à celles qui font partie du même environnement conceptuel, notamment dans l'élaboration de modèles d'intervention qui considèrent la complexité de la réalité sur laquelle porte l'intervention sociale ainsi que le caractère interactif des constituantes de la dite intervention :

"Les modèles proposés par la cybernétique, la théorie des systèmes, de la communication et des organisations, semblent répondre de façon plus appropriée à la complexité et à l'interaction des phénomènes qui font l'objet de notre intervention. Ils proposent au praticien des fonctions, des rôles et des instruments plus diversifiés que ceux impartis au clinicien-thérapeute de type traditionnel. La littérature professionnelle des récentes années rend compte d'un état de recherche et d'expérimentation - voire d'innovations - à cet égard. " 12 
Pour la compréhension d'une réalité complexe, l'auteure propose la théorie des systèmes, bien connue pour le potentiel qu'elle a d'intégrer les parties dans un tout - un système - qui, au demeurant, leur est irréductible. En outre, les modèles, produits aussi bien de la cybernétique que de la systémique, introduisent, selon la même auteure, une diversification des composantes de la pratique du travail social que l'on ne rencontre pas dans celui de type clinique. L'unité au sein de cette diversité souhaitée dans le travail social des années soixante-dix serait assurée, de surcroît, par la nécessité de la " convergence " postulée dès le départ de chacune des deux théories mentionnées ci-haut.

De même que Jeannine Godbout se prononce en faveur de la recherche de la diversité en travail social en recourant à des modèles issus de théories générales fondées sur la nécessité de la convergence, de même la Corporation des travailleurs sociaux du Québec retient-elle, de façon encore plus affirmative et manifeste, une perspective systémique dans sa définition de l'acte professionnel:

"L'objet de l'Acte professionnel du travailleur social est la Socialité, c'est-à-dire l'Équilibre dynamique dans les rapports entre les personnes et leur environnement immédiat ou médiat, dans une perspective de développement humain et social. „ ${ }^{13}$

Cette définition, empruntée à Jean-Pierre Duplantie et Jean-Bernard Robichaud qui proposaient, dès 1976, la socialité comme objet de la mission des services sociaux, ${ }^{14}$ fait de l'équilibre - notion centrale de la théorie des systèmes - l'objet de la pratique du travail social. En d'autres termes, la socialité en tant qu'équilibre (normatif il va de soi) serait, d'après eux, le point d'arrivée, le lieu de convergence de pratiques et d'acteurs qui, au départ, se différencient à plusieurs égards. ${ }^{15} \mathrm{On}$ voit donc comment la théorie des systèmes - qui, du reste, constitue leur cadre de référence théorique principal dans l'explication qu'ils font de la définition de la socialité, ${ }^{16}-$ s'avère une voie théorique fertile dans la recherche de l'organisation et de l'intégration des savoirs en travail social.

Sans être tout à fait explicites sur la place qu'occupe le paradigme systémique dans la formulation qu'ils font de l'approche intégrée dite conflictuelle en travail social, ou "approche structurelle", Maurice Moreau, Justin Lévesque et Jean Panet-Raymond font néanmoins usage de la notion de système dès l'énoncé du postulat de base de la dite approche : "[...] les rapports entre les systèmes sont considérés comme antagonistes, opposés et inégalitaires ". ${ }^{17}$ Même si les auteurs se démarquent de «l'approche intégrée consensuelle, basée sur la théorie générale des systèmes et sur les liens qui unissent les différents systèmes et les sous-systèmes entre eux ", 18 il n'en demeure pas moins que le postulat 
est énoncé en termes systémiques, avec la différence que la règle du conflit est substituée à celle du consensus. Or, cette substitution n'exclut pas une vision systémique des choses, tout comme d'ailleurs la vision systémique n'exclut d'aucune façon le conflit, mais en fait une possibilité théorique et un atout empirique dans l'action. Il nous apparaît donc que, là encore, la notion de système organise la connaissance et la pensée en travail social ce qui, par ailleurs, n'enlève rien à l'originalité certaine du produit, produit qui, à notre avis, représente une contribution importante dans cet effort qui tente de donner une cohérence au pluralisme qui caractérise les pratiques du travail social.

Notons au passage que l'attribut " structurel " que l'on accole à cette approche, tient de la notion de structure qui fait du reste partie de l'environnement théorique de la "systémique " ${ }^{19}$ et du " système social ". 20 Structure et système sont donc deux notions qui ne s'excluent pas et qui ont, lorsque réunies, une portée synthétisante sur la représentation de la réalité, notamment celle qui constitue l'objet du travail social. En effet, la synthèse que ces deux notions donnent à entendre confère aux constituantes de la dite réalité des caractères interactif et rétroactif, mais aussi le caractère irréductible de cette même réalité à ses constituantes. C'est ce qui amène les auteurs à poser que «dépendamment de la position dans l'organisation et la division du travail social, selon l'âge, le sexe et la classe sociale, les conditions de vie et de travail social sont en grande partie déterminantes de la santé physique et de la façon de penser, de sentir et d'agir des individus et des collectivités ". ${ }^{21}$ Une telle position met en évidence les caractères interactif et rétroactif de l'objet de la pratique et permet de franchir un pas de plus dans la recherche de la cohérence et de la synthèse par la voie d'une pratique intégrée.

Cette recherche de la synthèse est particulièrement frappante dans la formulation que fait Pierre Daher de l'intervention dite " de réseau ". 22 En effet, partant de la prémisse selon laquelle l'individu est porteur du "discours collectif de son réseau ", ${ }^{23}$ Daher souligne, de plus, que dans une pratique de réseau, "lorsque nous entrons en contact avec des individus, c'est à ce qu'il y a de collectif en eux que nous nous adressons ". ${ }^{24}$ Pour mieux rendre compte de ce caractère de totalité chez l'individu redevable à un tout plus grand qu'est son collectif, son réseau, l'auteur se sert de concepts systémiques en ajoutant : «[...] c'est-à-dire que nous les percevons [les individus] comme un sous-système du système global du réseau ". ${ }^{25}$ Tout se passe comme si la systémique était, pour l'auteur, l'ultime moyen pour représenter la place de l'individu dans le tout qui l'investit. Une telle représentation empêche de basculer dans un certain individualisme méthodologique que l'on attribue ordinairement au casework, comme elle permet de se démarquer de ce collectivisme où 
les protagonistes se méfient de l'individu perçu comme porteur d'une subjectivité qui rend inapte à voir les enjeux collectifs. Dans les circonstances, la systémique permet à l'auteur de maintenir la communication entre l'individu et son collectif d'appartenance, le réseau. Il contribue ainsi à mettre de l'avant une version particulière de la pratique sociale intégrée.

\title{
La recherche d'une méthodologie plurielle
}

La différenciation du champ du travail social se reconnaît de prime abord à la présence, dans ce même champ, de trois méthodes d'intervention (le travail social personnel ou casework, le travail social de groupe et l'organisation communautaire) pour approcher les situations problématiques qui appellent une intervention sociale. Ces méthodes d'approche sont devenues des méthodologies relativement autonomes par la force de la spécialisation et par l'impact des a priori idéologiques qui orientent objectifs et actions. Néanmoins, nous assistons depuis quelque temps à un effort renouvelé vers l'articulation d'une méthodologie plurielle et intégrée, comme le reflètent clairement certains travaux récents. Nombreux sont les travaux significatifs élaborés dans cette perspective. Cependant, nous nous limiterons ici à ceux dont le procédé d'intégration nous paraît le plus explicité. Par exemple, Maurice Moreau et ses collaborateurs utilisent le procédé suivant dans leur conception d'une méthodologie plurielle :

\begin{abstract}
«À partir d'une analyse qui identifie l'importance des facteurs biologiques, psychologiques, idéologiques, culturels, sociaux, économiques et politiques en présence dans une situation-problème, l'intervenante définit un plan d'intervention comprenant une coordination et une continuité d'action à différents niveaux. Selon le problème, l'action pourra d'abord être centrée soit au niveau personnel/interpersonnel, soit au niveau institutionnel/communautaire et évoluer selon un continuum. Ou encore l'intervention se fera parallèlement à plus d'un niveau [...]. L'intervenante sociale formée à une pratique structurelle aura donc développé des connaissances analytiques et méthodologiques lui permettant d'évaluer une situation, de définir un plan d'intervention portant sur un ensemble de facteurs et à différents niveaux. \ 26
\end{abstract}

D'une pratique marquée par des discontinuités redevables au découpage multiple de l'objet, en travail social, découpage issu des impératifs techniques et aussi idéologiques comme nous le verrons à la prochaine rubrique, ces auteurs nous convient désormais à la continuité en plaçant les gestes du praticien sur un même continuum plutôt que de les répartir sur un ensemble d'acteurs. Dès lors, le praticien en travail social aura à 
assurer une coordination de ses gestes en les intégrant au tout conçu à l'avance et duquel les gestes en question sont redevables. Ceci implique que le praticien habilité à utiliser cette méthodologie plurielle devra recevoir une formation en conséquence, c'est-à-dire intégrée plutôt qu'éclatée, où chacune des parties prétend à une autonomie propre et à une singularité, tant de l'objet d'intervention que des manières de faire pour l'appréhender et le traiter. Les frontières entre l'individu, le groupe et la communauté s'estompent au profit d'un renouement avec une nouvelle unité à l'instar, faut-il le souligner, de celle que traduisent les trois auteurs dans leur enseignement du travail social.

Un autre courant, l'intervention dite " de réseau ", prône une méthodologie intégrée en travail social, mais son procédé s'articule autour de la notion de réseau plutôt que de celle de structure. À cet égard, les écrits de Claude Brodeur, Jacques Alary et Pierre Daher sont ici mis à contribution pour repérer ce procédé, ne serait-ce que dans son principe. Tout en prenant garde de ne pas trahir ou biaiser leur pensée, on peut dire, dès l'abord, que l'approche de réseau pose que l'individu est redevable aux réseaux ou " petits collectifs " 27 auxquels il appartient, tout comme ces derniers sont inconcevables sans les individus qui les constituent. En soi, cette prémisse n'a rien de spécifique à cette approche. Elle permet cependant de se représenter le champ de l'intervention comme un tout complexe, une combinatoire de réseaux qui fait que
"la demande [de service] qui s'exprime lors d'un contact avec un établissement de travail social n'est pas le fait d'un individu, mais celui d'un collectif [réseau], et que, d'autre part, la réponse souhaitée n'est pas une prise en charge de caractère institutionnel et professionnel, mais plutôt un support technique dans une démarche de renvoi à son propre savoir et de mobilisation de ses propres ressources ". ${ }^{28}$

Le requérant n'est pas isolé de son réseau d'appartenance : les deux sont interdépendants et appellent donc une méthodologie d'analyse et d'intervention qui les engage l'un dans l'autre dès l'instant où l'intervenant - que l'on présuppose être formé en conséquence c'est-à-dire à une méthodologie plurielle - entre en jeu. Le support technique de ce dernier n'est pas plus destiné au requérant qu'au réseau auquel celui-ci est redevable.

On aura compris, à la suite de cette brève analyse de l'intervention dite de réseau, qu'il s'agit là d'une approche holistique dans la mesure où la méthodologie de base préconise un "recentrage » de l'expression singulière d'un problème dans la réalité collective dont elle provient, tout en réactivant le réseau significatif pour la recherche de solution. Pierre Daher constate en effet, qu'en "refusant de "focaliser" ou d'axer notre intervention sur les problèmes de Marguerite, nous avons en 
quelque sorte déjoué la stratégie du milieu qui en faisait son porteparole et avons forcé celui-ci à prendre conscience de ses vrais problèmes, ouvrant ainsi la voie à l'élaboration de solutions vraiment efficaces. „ ${ }^{29}$

\section{Des synthèses idéologiques dans les pratiques sociales intégrées}

II nous faut remonter à 1976 pour repérer un discours qui propose, en l'articulant, un pluralisme idéologique pour le travail social québécois. En effet, Jean-Pierre Duplantie et Jean-Bernard Robichaud présentent, selon le procédé idéal-typique, deux modèles d'intervention dont l'un, le modèle intégrationniste, se situe à l'extrême de l'autre: le modèle conflictuel. ${ }^{30}$ Ils posent en même temps que "la réalité concrète de l'intervention représentée par ces types idéaux ne se situe jamais à l'un des deux extrêmes [...]", 31 c'est-à-dire que la pratique du travail social n'asseoit pas sa normativité sur des pôles extrêmes considérés comme étant dogmatiques par les mêmes auteurs, mais plutôt entre les deux extrêmes, espace qu'ils envisagent comme un continuum sur lequel peut s'étaler aussi bien l'intervention individuelle que l'intervention collective. C'est ce qui les amène à poser qu'il y a des interventions sur le plan individuel qui peuvent être de type conflictuel, comme d'autres qui peuvent être de type intégrationniste ", 32 tout comme "il est également possible de démontrer que l'intervention collective peut être soit intégrationniste, soit conflictuelle ${ }^{33}$

Repoussant le dogmatisme et préconisant le réalisme dans l'intervention, ils admettent doncl'usage possible des deux vecteurs normatifs (conflictuel et intégrationniste), aussi bien dans l'intervention sociale individuelle que collective; ce faisant, ils prennent le contrepied des tenants d'une représentation du travail social dans laquelle l'intégrationnisme est caractéristique de l'intervention individuelle, tandis que le conflit serait l'apanage de l'intervention collective. En outre, en évacuant le dogmatisme qu'ils rattachent à l'extrémisme normatif, ces auteurs ont une représentation de l'intervention sociale dans laquelle l'intégration et le conflit n'apparaissent plus comme des antinomies, mais bien plus comme des vecteurs normatifs différents, dirigés toutefois vers la recherche d'un "état d'équilibre dynamique entre les systèmes ou sous-systèmes d'un système " ${ }^{34}$

Ainsi sommes-nous en présence de conditions normatives de pratiques sociales formulées de telle façon que les divergences dont elles sont porteuses s'avèrent, au fil de l'action, un prélude à une possible convergence, probablement ce en quoi consiste la "socialité». En ce sens, le procédé théorique emprunté pour étayer le concept de socialité porte 
en lui les germes d'une pratique en travail social dont les parties, si normatives soient-elles, sont au service du tout; bref, d'une pratique intégrée.

Notons au passage que l'usage intensif que Duplantie et Robichaud font de la systémique pour étayer leur représentation, notamment de la pratique sociale, a un effet d'érosion sur les positions idéologiques aiguës et cristallisées que l'on rencontre souvent dans la pratique sociale, effet légitimé de surcroît par le recours au réalisme aux dépens du dogmatisme.

Longtemps, l'intervention collective, et notamment, celle de type militant, a fait taire, ou du moins mis en veilleuse, la dimension individuelle du travail social collectif. Une telle attitude est reliée, en partie, à des analyses sociales de problèmes de société qui imputent les causes à la structure même des rapports sociaux, excluant du même coup l'imputabilité à l'individu, dans le diagnostic forcément collectif des mêmes problèmes, et préconisant des interventions du même ordre. Il s'ensuit une pratique militante où l'intervenant a à vivre une certaine abnégation de soi, comme le constatent, avec justesse d'ailleurs, les porte-parole du collectif québécois de conscientisation lorsqu'ils écrivent que "les exigences imposées dans la vie militante ne tiennent pas suffisamment compte du vécu privé " ${ }^{35}$ et ajoutent que "trop souvent aussi jusqu'à maintenant, des groupes de lutte ont fonctionné uniquement en fonction des tâches à accomplir sans accorder trop d'attention aux personnes en cause $[\ldots]]^{36}{ }^{36}$ Le sujet, conscience individuelle, longtemps évacué de l'univers militant et de ses préoccupations centrales pour son propre devenir collectif, devra donc désormais retenir l'attention du milieu militant où « un effort semble s'imposer pour remettre en valeur le pôle personnel \%. ${ }^{37}$ La réconciliation du sujet individuel avec le sujet collectif devrait, selon le même milieu, viser " un équilibre heureux entre l'attention accordée à la démarche collective et celle accordée aux personnes qui vivent l'action ". ${ }^{38}$

Dès lors, en restituant à l'individualité sa place dans le collectif qu'elle contribue à former, la nouvelle pratique militante, notamment celle de la conscientisation, participe, à sa manière bien entendu, au courant de la pratique sociale intégrée. À sa manière, disons-nous, puisqu'il ne s'agit pas, pour les auteurs, de renouer avec un "certain psychologisme" dont ils prennent la peine de se démarquer, ${ }^{39}$ mais plutôt de permettre à l'individualité de s'exprimer sans " perdre de vue ce pôle collectif " ${ }^{40}$ sans lequel un "des axes de conscientisation " 41 "personnel $\leftrightarrow$ collectif» 42 - fait défaut de même qu'une "véritable démarche de conscientisation $» .{ }^{43}$ 
On aura compris que le discours militant de la pratique sociale réhabilite l'individu et son vécu propre après les avoir pratiquement conjurés de l'analyse sociale. Toutefois, cette réhabilitation est assortie de conditions, notamment celle qui pose que l'individu ne peut connaître une véritable libération que dans la mesure où entre la "conscience individuelle" ${ }^{44}$ et la "conscience communautaire $\leftrightarrow$ de classe", 45 un "processus dialectique" s'instaure, aussi bien au plan de la réflexion que de l'action. ${ }^{46}$ Cette recherche du tout par l'unité des contraires que toute dialectique présuppose inscrit, selon nous, les nouvelles pratiques de conscientisation telles que formulées par le collectif Ampleman, Doré et al. dans le courant des pratiques intégrées. Toutefois, le collectif ne fait pas qu'additionner la dimension de la "personne», comme le ferait une sédimentation, mais il lui procure une légitimité qui tient du discours propre aux pratiques de conscientisation, discours qui, selon le même collectif, pose que "tous les individus ne sont pas au même point : chacun est à un niveau de conscience donné, chacun a ses habiletés, son bagage, ses désirs d'épanouissement particuliers 1.47 Cette légitimité oblige ensuite le collectif à réaménager une place aux considérations personnelles dans son projet collectif : "l'organisation doit donc, dans l'établissement collectif de ses priorités, tenir compte des besoins des membres, de leur disponibilité et, dans la répartition des tâches, de leurs goûts et de leurs capacités ". 48

Cette recherche de cohésion et de synthèse, de même que de rapprochement de l'individu à son collectif d'appartenance et inversement, bref cette proposition d'une vue d'ensemble aura l'effet d'éroder les susceptibilités des idéologies collectiviste et individualiste. C'est sans doute sous cet angle que se situe l'apport principal du collectif québécois de conscientisation à la mise en forme d'une pratique sociale intégrée.

\section{Conclusion}

Nous posions comme proposition de départ que les pratiques sociales intégrées qui se développent dans le champ du travail social traduisent un mouvement de recherche de la convergence et de la synthèse d'éléments constitutifs et différenciés du champ en question. Nous posions aussi que ce travail de (re)constitution d'une identité plurielle au sein du travail social s'effectue selon un procédé tout aussi pluriel. Les quelques écrits auxquels nous avons soumis cette proposition, afin de lui trouver échos et fondements, se sont avérés porteurs de ce mouvement de recherche de la convergence et de la synthèse. Ces 
discours, dont chacun fait autorité au sein du milieu du travail social québécois pourraient, il va sans dire, être scrutés davantage dans le cadre d'une étude plus large ; cela permettrait de dégager de façon encore plus probante cette recherche de la convergence et de la synthèse qui, d'ores et déjà, semble esquisser, au fil du mouvement et à l'instar du procédé déployé, une identité plurielle pour le travail social.

En attendant ce travail de profondeur, limitons-nous à constater qu'à ces discours correspondent des pratiques. Ces deux éléments ont un effet réorganisateur certain sur les représentations traditionnelles ou institutionnalisées du travail social, en y atténuant les divisions par méthodes et la cristallisation des positions idéologiques. Toutefois, ces discours et ces pratiques ne débouchent pas encore sur une voie convergente en travail social, voie à partir de laquelle des angles d'attaque pourraient se développer, s'interpeller et vers laquelle un questionnement serait adressé. Ce sont plutôt des véhicules pouvant conduire à une problématique de la connaissance propre au travail social. Car, pour nous, cette recherche de la synthèse et de la convergence qui se dégage des propositions principales de chacune des pratiques sociales intégrées que nous avons passées en revue soulève, par-delà des réponses immédiates qui assurent la survie du champ, un questionnement relatif à l'unité paradigmatique en travail social et à la connaissance pertinente à l'étaiement de cette unité.

Quoi qu'il en soit, telles que nous les avons appréhendées à travers certains écrits, les pratiques sociales intégrées actuelles, au Québec en l'occurrence, constituent un véritable tournant de maturation du travail social et, de ce fait, elles invitent la réflexion à s'engager dans une problématique de la connaissance en ce champ, prélude à son unité paradigmatique. Le dialogue entre les pratiques sociales intégrées, nonobstant la forme qu'il prendra, nous apparaît dès lors nécessaire. À cet égard, l'expérience d'échange entre des tenants de différentes pratiques intégrées, qui a été amorcée en 1984 dans le cadre des travaux du Groupe d'échange sur les approches intégrées (le GEAI, auquel nous faisions allusion en introduction), constitue un point de départ. La multiplication d'échanges de ce genre contribuera sans doute à mieux approfondir les connaissances en travail social et, par voie de conséquence, à délimiter le lieu de convergence de ces connaissances. 


\section{Notes et références}

1 L'auteure tient à remercier Belhassen Redjeb, professeur au Cégep de LévisLauzon, pour la lecture attentive et commentée qu'il a faite de la version préliminaire du présent article.

2 Mary Richmond, Social Diagnosis, (1917), citée dans: Harry SPECHT et Anne VICKERAY, Integrating Social Work Methods, London, Allen \& Unwin, 1977, p. 15.

3 Harry SPECHT et Anne Vickeray, Id.

4 Francine Ouellet, "Une conception de la formation et de l'évaluation en service social ", dans : Justin LÉVESQUE et. al., (dir.) La formation en travail social au Québec: d'une école à l'autre, Montréal, Regroupement des unités de formation en travail social du Québec, 1984 : 69-90.

5 Id., p. 69.

6 Id., p. 70.

7 Francis P. PURCELL et Harry SPECHT, "The House on Sixth Street ", Social Work, vol. 10, no 4, $1965: 65-76$. Cette étude de cas a été reprise, entre autres, la même année par la N.A.S.W., puis en 1977 dans: Integrating Social Work Methods, op. cit., de même qu'en 1984 par Beulah COMPTON et Burt GaLAWAY dans : Social Work Processes, Homewood (III.), Dorsey Press.

${ }^{8}$ Alice Home, Marcelle Laforest et Pierre Tremblay, "Une analyse de l'évolution de la pratique du service social en contexte organisationnel au Québec", Intervention, no 58, été $1980: 4-12$. Voir aussi : Pierre TremblaY, "Une pratique alternative", dans: Domination technocratique et pratique alternative dans les services sociaux, essai non publié, Centre de documentation, École de service social, Université Laval, 1986 : 99-134.

9 Jeannine Godbout, "Les nouveaux défis de la pratique professionnelle ", Le Travailleur social, vol. 40, no 2, mai 1972: 68-75.

10 Id., p. 68.

11 lbid.

12 Id., p. 75.

13 "Définition de l'Acte professionnel du travailleur social ", tel qu'adopté par le Bureau de direction de la Corporation professionnelle des travailleurs sociaux du Québec le 24 février 1984.

14 Jean-Pierre Duplantie et Jean-Bernard Robichaud, "La mission des Centres de services sociaux : la socialité par les services sociaux spécialisés ", Intervention, no 47, automne 1976 : 3-19.

15 lbid.

16 Ibid.

17 Maurice Moreau, Justin LÉvesque et Jean PANet-Raymond, "L'approche structurelle : un défi pour les éducateurs en travail social ", dans La formation en travail social au Québec: d'une école à l'autre", op. cit., p. 92.

18 Ibid.

19 Daniel Durand, La systémique, Paris, Presses universitaires de France. Collection Que sais-je ?, 1979: 32-36. 
20 Talcott Parsons, dans sa théorie générale de l'action, fait de la structure une des trois conditions d'un système. Voir: Talcott ParSOnS, The Social System, New York, Free Press, 1951.

21 Maurice Moreau et al., op. cit., p. 92.

22 Pierre DAHER, "Les pratiques de réseaux : recherche-action", Intervention, no 58 , été $1980: 13-23$.

23 Id., p. 22.

24 Ibid.

25 Ibid.

26 Maurice Moreau et al., op. cit., p. 92-93.

27 Jacques ALARY, "Les antécédents de "l'approche réseau" en service social", Service social, vol. 29, n० 3, 1980, p. 267.

28 Id., p. 269.

29 Pierre DAHER, "Histoire d'une intervention de réseaux ", Service social, vol. 29, n० 3, 1980, p. 302.

30 Jean-Pierre Duplantie et Jean-Bernard RobiChaud, "La mission des centres de services sociaux... ", op. cit., p. 12.

31 Ibid.

32 Ibid.

33 Ibid.

34 Id., pp. 17-18.

35 Gisèle Ampleman, Gérald Doré, et al., Pratiques de conscientisation : expériences d'éducation populaire au Québec, Montréal, Nouvelle Optique, 1983, p. 276.

36 Id., pp. 276-277.

37 Id., p. 277.

38 Ibid.

39 Id., p. 279.

40 Id., p. 281.

41 Ibid.

42 Ibid.

43 Id., p. 278.

44 Id., p. 274.

45 Ibid.

46 Ibid.

47 Id., p. 277.

48 Ibid. 